The Astrophysical JourNAL, 539:933-943, 2000 August 20

(C) 2000. The American Astronomical Society. All rights reserved. Printed in U.S.A.

\title{
EMERGENCE OF EMBEDDED MAGNETIC FLUX AND ITS CONNECTIONS TO THE SOLAR DYNAMO
}

\author{
TZIHONG CHIUEH \\ Department of Physics, National Taiwan University, Taipei, Taiwan, and Institute of Astronomy and Astrophysics, \\ Academia Sinica, Taipei, Taiwan; chiuehth@phys.ntu.edu.tw \\ Received 1997 September 9; accepted 2000 February 11
}

\begin{abstract}
A strong $\left(\sim f e w \times 10^{5} \mathrm{G}\right)$ and slightly twisted magnetic field (of flux $\left.10^{23} \mathrm{Mx}\right)$ can be stored in a dynamo flux tube within the overshoot region provided that the tube has a slightly lower temperature than the background gas, which has a stratification profile $\gamma(d \ln \rho / d \ln P)-1 \geq B^{2} / 8 \pi P$, where $P, \rho, B$ and $\gamma$ are the gas pressure, density, magnetic field strength, and adiabatic index. Pumped by the intense vortical downdraft, the outer layer of dynamo flux tube is proposed to possess opposite magnetic helicity from that in the tube interior, as a direct result of the conservation of magnetic helicity. When the fieldline twist in the outer layer exceeds some threshold, we show that the helicity-reversal layer can be subject to MHD instabilities that trigger partial flux eruption. In the nonlinear stage, flux ropes of $10^{22}$ Mx with magnetic helicity of a definite sign are expected to be ejected into the convection zone, leaving magnetic helicity of the opposite sign to accumulate in the main flux tube. Such a helicity removal mechanism breaks the conservation of magnetic helicity within the dynamo flux tube and can circumvent the recently discovered difficulty (the so-called $\alpha$ quench) for the generation of large-scale fields. We argue that the dynamo $\alpha$ is likely to resume a value of the same order as that of the kinematic turbulent dynamo. We further suggest that the helical flux expulsion is an indispensable component for the solar dynamo to operate properly. A natural prediction of this scenario is that strong sunspots in the same hemisphere should possess magnetic helicity of the same sign, regardless of the solar cycles. Specifically, the helical field lines in strong sunspots should obey the left-hand rule in the northern hemisphere and the right-hand rule in the southern hemisphere.

Subject headings: MHD - Sun: interiors
\end{abstract}

\section{INTRODUCTION}

The solar magnetic fields are believed to be amplified by the combination of solar differential rotation and helical turbulence. Such an amplification mechanism is known as the $\alpha-\Omega$ dynamo (Parker 1979; Moffatt 1978). Although this mechanism needs turbulence, the presence of vigorous turbulence can, at the same time, scramble the magnetic fields to the extent that the coherent magnetic fields as observed at the sunspots are difficult to be produced. In circumventing the contradictory needs for the presence of turbulence, it has been suggested that the site for dynamo action should be located immediately beneath the convection zone in the stably stratified, overshoot region (Spiegel \& Weiss 1980; Galloway \& Weiss 1981). This convenient location makes the magnetic fields on one hand extract the partial action of turbulence and on the other avoid the fatal disruption at the generation site.

The above scenario, although it may solve the disruption problem, must face another problem; that is, how the magnetic fields can be stored and amplified at this convenient location for a period on the order of several years. From the considerations of pressure balance, one expects the storage of magnetic fields to be nontrivial. The magnetic flux tube must have a lower gas pressure, which normally implies a lower density; if so, one then has a situation where a light magnetized bubble is immersed within a heavy fluid against the gravity. The light bubble cannot be in equilibrium with the background gases but to float upward into the convection zone, making the storage of the flux tube at this convenient location difficult.

Convective turbulence, exerting turbulent pressure on the magnetic flux in the overshoot region, may be a candidate to solve the flux the storage problem. In this scenario, there exists a maximum field strength below which the flux tube may be stored. Equipartition between the magnetic energy density and the kinetic energy density of convective turbulence defines this maximum field strength. Such an equipartition field strength amounts to $10^{4} \mathrm{G}$. For the $10^{22} \mathrm{Mx}$ magnetic flux, estimated from a large active region, the flux tube minor radius is about $6 \times 10^{3} \mathrm{~km}$ in the storage region. However, investigations on the emergence of flux tubes have found that a field strength as low as $10^{4} \mathrm{G}$ in the overshoot region can never yield any coherent sunspot as observed on the solar surface, nor can it emerge at low latitudes (Choudhuri \& Gilman 1987; Fan, Fisher, \& Deluca 1993; Moreno-Insertis \& Ferriz-Mas 1992; D'Silva \& Choudhuri 1993). It is hence concluded that a field strength as large as $10^{5} \mathrm{G}$ must be needed (Choudhuri \& Gilman 1987; Schussler et al. 1994; Caligari, MorenoInsertis, \& Schussler 1995). One of the implications for such a strong field is that convective turbulence cannot play the primary role in confining the flux tube.

In view of the possible failure of turbulence confinement, one plausible solution to the flux storage problem can be that the tube has a lower overall temperature, instead of lower overall density, so that a lower gas pressure can be achieved (Moreno-Insertis, Schussler, \& Ferriz-Mas 1992; Ferriz-Mas 1996). Such a solution must again face another problem. That is, thermal diffusivity in the solar interior is rather efficient and a discontinuous change of temperature across the flux tube boundary should be difficult to sustain. Actually, one may circumvent this problem by allowing the flux tube to have a thick thermal blanket, within which the temperature gradually rises from the interior to match onto 
the ambient temperature outside. A possible cause for a slightly lower overall temperature within the flux tube is the reduced electron heat conductivity across the magnetic fields. In the overshoot region where the mass density is about $2 \mathrm{~g} \mathrm{~cm}^{-3}$ and temperature about $10^{6}$ degrees, the electron conduction is the dominant heat transport mechanism. Hence, any reduction in the electron cross-field mobility can certainly reduce the collisional mean free path and modify the temperature profile within the flux tube. In fact, the amount of temperature reduction required to house a neutrally buoyant flux tube is tiny, on the order of $\beta^{-1}$, where $\beta$ is the ratio of plasma thermal pressure to the magnetic pressure. For example, a few $\times 10^{5} \mathrm{G}$ field will have a plasma $\beta$ about $10^{5}$. Therefore the needed reduction of the electron mean free path is correspondingly small.

Viable equilibrium configurations for the flux tube may also need to meet additional requirements apart from the smooth variation of temperature across the flux tube. It has been suggested that twisted fields (i.e., nonvanishing poloidal fields) in a toroidal flux tube can create stabilizing magnetic tension in retaining the integrity of a flux tube when the tube rises through the turbulent convection zone (Cattaneo, Chiueh, \& Hughes 1990a, 1990b; MorenoInsertis \& Emonet 1996; Emonet \& Moreno-Insertis 1998; Dorch \& Nordlund 1998; Hughes et al. 1998; Fan, Zweibel, \& Lantz 1998a; Fan et al. 1998b, 1999). For a simplified geometry of a planar flux layer, two-dimensional calculations conducted by Cattaneo et al. (1990a) show that disruptive instabilities tend to occur above a particular flux surface, at which the field lines reverse the twist, thereby peeling off the upper part of the flux layer and leaving the main layer intact. Once the fields are reconnected and rise, they may emerge as discrete twisted flux ropes. It was further proposed by Cattaneo et al. (1990b) that in the solar active phase this surface can be located well within the tube, and in the quiet phase near the outer edge of the tube. Such a difference may explain why the coherent sunspots appear so abundantly in the active phase and are nearly absent in the quiet phase. The presence of both toroidal and poloidal fields in the escaped magnetic flux also explains why the sunspot magnetic fields are twisted. In a qualitative sense, the scenario suggested by Cattaneo et al. offers a possible explanation for the magnetic features during half a solar cycle without invoking any change of turbulence in the convection zone. It however leaves the issue of electric current variations within the flux tube to the problem of dynamo waves (Parker 1993; MacGregor \& Charbonneau 1997). In view of the recent detection of a sharp differential rotation in the overshoot region (Kosovichev et al. 1997), we propose a variation of the model of Cattaneo et al. (1990a), in that the toroidal field reverses the sign within the flux tube at some flux surface but the poloidal field remains of the same sign throughout the tube. This arrangement renders the outer layer of the tube to have an opposite field-line twist to that well within the tube. (This opposite twist is explained, in the present model, to be derived from the helicity of the turbulent downdraft in the convection zone.) Much like the model of Cattaneo et al., it is also the outer layer of the tube that will be shown to become unstable and is expected to get peeled off and emerges to the solar surface. Therefore, the additional conditions to be imposed on the flux tube equilibrium is that there should exist both toroidal and poloidal fields to yield the twisted field lines, and that there also exists a region of toroidal-field reversal, in which the field-line twist is relatively strong. The purpose of the present work are first to consider a more realistic situation, where the magnetic flux is confined within a tube rather than within a planar layer (Cattaneo et al. 1990a), and second to expand the proposal of Cattaneo et al. (1990b) to a deeper scope in connection to the operation of the solar dynamo.

In the past, various models have been proposed to address how the magnetic flux may emerge from the solar interior. The ones, which have received appreciable attention due to their simplicity, work under the assumption of the thin-tube approximation, where the internal structures of flux tube are ignored (Spruit 1981). Such models predict that the toroidal flux tube will undergo the undular instability when the field strength is sufficiently strong (van Ballegooijen 1982; Schmitt, Rosner, \& Bohn 1984), and a segment of it can emerge to the solar surface in an $\Omega$ shape without suffering from internal disruption (Caligari et al. 1995; Fan et al. 1998). This picture is in marked contrast to the aforementioned model of Cattaneo et al., where the flux tube undergoes internal disruption and only the outer layer of the flux tube is dispatched from the main tube; the remaining magnetic flux retained in the main tube may provide the seed field for the dynamo action to continue.

In the conventional picture of the $\alpha-\Omega$ dynamo, the toroidal fields are generated by the shearing of the poloidal fields as a result of differential rotation at the overshoot region. This aspect of dynamo model has recently gained a strong support from the solar seismology observations that indicate the existence of a sharp shear layer at the aforementioned location (Kosovichev et al. 1997). However, it is the regeneration of the poloidal fields that poses the major challenge for the solar dynamo action. On one hand, the dynamo requires helical turbulence to give rise to the twist and fold motion of field lines, in which processes the poloidal fields are generated. On the other hand, it also requires the ohmic dissipation to break the field lines so that the large-scale magnetic fields can be built up. However, in a closed volume, a well-known theorem exists, stating that the magnetic helicity, measuring the total linkage of field lines, is a nearly perfectly conserved quantity in a highly conducting fluid (Moffatt 1978). (The magnetic helicity is defined to be $\int \boldsymbol{A} \cdot \boldsymbol{B} d^{3} x$, where $\boldsymbol{B}$ and $\boldsymbol{A}$ are the magnetic field and the associated vector potential, respectively.) Some years ago, it has been shown that due to the conservation or magnetic helicity in a closed volume, the maximum energy of the large-scale fields generated by the dynamo action cannot exceed $R_{m}^{-1}$ of the turbulence energy, where $R_{m}$ is the magnetic Reynolds number (Gruzinov \& Diamond 1994; Cattaneo 1994; Cattaneo \& Vainshtein 1991). This small factor $R_{m}^{-1}$ associated with the large-scale field energy can simply be estimated from a generalization of the Zeldovich theorem for two-dimensional MHD (Zeldovich 1957; Cattaneo \& Vainshtein 1991). It is due to this small factor $R_{m}^{-1}$ that the mean-field turbulent dynamo practically fails to operate in a highly conducting plasma. In order words, the feedback of the small-scale turbulent magnetic field efficiently quenches the dynamo coefficient $\alpha$.

Nevertheless, this recently discovered "dynamo-quench" mechanism can become invalid when the dynamo operates in an open system where the magnetic flux can escape. In particular, when only the flux with magnetic helicity of a particular sign can escape from the dynamo region, the magnetic helicity in that region will not be conserved. In 
this report, we wish to show that the dynamo flux tube in the overshoot region can become unstable when the magnetic field twist has a sufficiently large gradient; such an instability is likely to give rise to escape of the field lines possessing only one sign of twist, i.e., one sign of magnetic helicity. This flux expulsion mechanism leads thus to ejection of magnetic helicity, thereby invalidating the "dynamo-quench" mechanism. We shall also emphasize that if the large-scale magnetic flux is indeed to be generated within the Sun, the ejection of magnetic helicity appears to be inevitable, and such an ejection mechanism should be operative simultaneously with the usual $\alpha-\Omega$ dynamo. In other words, the flux emergence, although intuitively not obvious, assists the operation of dynamo, rather than simply suppressing the dynamo as conventionally thought (Parker 1979; Noyes, Weiss, \& Vaughan 1984; Hughes \& Proctor 1988).

Since the solar dynamo is a complicated problem, the present work will not address the simultaneous operations of flux eruption and $\alpha-\Omega$ mechanism. We shall avoid the detailed physics of the dynamo waves and instead confine ourselves to exploring how to create a finite $\alpha$ for the $\alpha-\Omega$ dynamo in connection to the partially unstable flux tube. To this aim, we will first show that, much like the planar case, the plasma can be unstable beyond the flux surface where the twist reverses, provided that the local twist exceeds some threshold strength. An example of the equilibrium flux tube with opposite twists in a stably stratified background gas is given in $\S 2$. In $\S 3$, the stability analysis of this MHD equilibrium is presented. Detailed discussions on the implications of this stability to the operation of solar dynamo are presented in $\S 4$. Our conclusion is given in $\S 5$. In the Appendix, we show some technical details for the derivations presented in $\S 3$.

\section{EQUILIBRIUM FLUX TUBE}

If the aspect ratio of the toroidal flux tube is small, i.e., a slender tube, we may ignore the curvature around the major axis for the flux tube equilibrium. It is thus sufficient to consider a two-dimensional flux tube equilibrium. (Toroidal magnetic tension and solar rotation have been included for the construction of an embedded flux tube; for example, Caligari et al. 1995, but we ignore these effects for simplicity.) In this simple model, we assume the cross section of the flux tube to be circular, despite the fact that the background gases are stratified. We let the background gas pressure be $P_{0}$, and the gas pressure in the flux tube can be decomposed into two parts $P=P_{0}(z)+P_{1}(r)$. Here $r$ refers to the axial distance from the center of the flux tube. The force balance equation can be written as

$$
\hat{\boldsymbol{r}} \frac{d}{d r}\left(\frac{B_{y}^{2}+B_{\theta}^{2}}{8 \pi}+P_{1}\right)+\hat{z} \frac{d P_{0}}{d z}=-\hat{\boldsymbol{r}} \frac{B_{\theta}^{2}}{4 \pi r}-\hat{z} \rho g,
$$

where $y$ and $z$ are along the toroidal direction and against the gravity, respectively. For simplicity, we further assume that the plasma density $\rho$ inside the flux tube is the same as that in the background, i.e., a buoyantly neutral tube. Canceling the gravity with the background pressure force, equation (1) can be solved formally as

$$
B_{y}^{2}+B_{\theta}^{2}+8 \pi P_{1}=-2 \int_{0}^{r} \frac{B_{\theta}^{2}}{r} d r+C,
$$

where $C$ is an integration constant. Outside the flux tube, we require $B_{y}, B_{\theta}$, and $P_{1}$ to vanish. Thus, we choose $C$ such that

$$
P_{1}=-\frac{B_{y}^{2}+B_{\theta}^{2}}{8 \pi}+\int_{r}^{\infty} \frac{B_{\theta}^{2}}{4 \pi r} d r .
$$

There are only few constraints to be imposed on the viable flux-tube equilibrium, and hence the equilibrium is relatively free to choose. The constraint mentioned in the last section is that $B_{y}$ reverses the sign somewhere in the flux tube; in addition we need $P_{1}$ given by equation (3) to yield a positive gas pressure $P$. The second constraint can be easily satisfied since the plasma within the flux tube is of high $\beta$ and $P_{1} / P=O\left(\beta^{-1}\right)$. There is one more constraint that has not yet been mentioned, and it is the conservation of magnetic helicity. In other words, the magnetic helicity retained in the main tube should have a magnitude equal to the accumulated magnetic helicity already ejected, but with an opposite sign. As the magnetic helicity in the outer layer of the tube, which is directly derived from the helical convective turbulence, has quite a different origin from that well inside the main tube, the outer layer should contain a separate component of $B_{p}$. Conservation of magnetic helicity will allow us to estimate the relative strengths of the two components of $B_{p}$, and this will be carried out in $\S 4.4$.

The simplest choice for such an equilibrium flux tube can be

$$
\begin{gathered}
B_{y}=B_{0}\left(1-r^{2}\right)\left(1-\frac{r^{2}}{a^{2}}\right) \\
B_{\theta}=2 h B_{0} r(1-r)\left\{1+\kappa e^{-[2(r-1) /(1-a)]^{2}}\right\},
\end{gathered}
$$

and $\boldsymbol{B}=0$ for $r \geq 1$. Here $a<1, B_{0}$ is the toroidal field strength at $r=0$, and $h$ characterizes the strength of $B_{\theta}$ relative to $B_{y}$ in the main tube. The poloidal field $B_{\theta}$ contains two components; one is confined in an outer layer which derives itself from the helicity pumping of the turbulent downdraft from the convection zone, whereas the other permeates throughout the whole tube. The relative strength of the two $B_{p}$ components is characterized by the parameter $\kappa$. Such a choice for the equilibrium field ensures that the total field vanishes at $r=1$ and $B_{y}$ reverses the sign at $r=a$. In particular, it should be noted that the field lines outside $r=a$ have an opposite twist to that inside. This equilibrium configuration has continuous gas pressure (cf. eq. [3]) and magnetic field, hence yielding no current sheets anywhere.

On the other hand, outside the flux tube, the background pressure $P_{0}$ and density $\rho$ vary with height as

$$
\begin{gathered}
P_{0}=\frac{\beta B_{0}^{2}}{8 \pi}(1-\epsilon z)^{1+1 / m}, \\
\rho=\frac{\epsilon \beta B_{0}^{2}(1+m)}{8 \pi g m}(1-\epsilon z)^{1 / m},
\end{gathered}
$$

where we have assumed that the ambient equilibrium satisfies $P_{0} \propto \rho^{1+m}$ and $\epsilon$ can be regarded as the inverse temperature scale height. The parameter $\beta$ represents the plasma $\beta$ at the tube center. The convective stability of the ambient gases is ensured when $\gamma \geq 1+m$, where $\gamma$ is the index of adiabaticity. With an embedded flux, we need

$$
(\gamma-1-m) / \gamma \geq O\left(\beta^{-1}\right),
$$


to ensure the stability of the main flux tube, as shown in the next section. Thus the introduction of a flux tube yields only a small perturbation, of order $\beta^{-1}$, in the energy density to the ambient gas. Take a realistic numerical example. In $\S 4$, we will give an estimate for the field strength $\left(B_{0} \sim 5 \times 10^{5}\right.$ G) and magnetic flux $\left(\sim 10^{23} \mathrm{Mx}\right)$ in the dynamo flux tube. It follows that the average $\beta \sim 10^{5}$. In addition, the radius of the flux tube will also be estimated to be about $3000 \mathrm{~km}$. If we take the temperature scale height of the background gas to be $10^{5} \mathrm{~km}$, giving $\epsilon \sim 3 \times 10^{-2}$. The quantity $(\beta \epsilon)^{-1} \sim 3 \times 10^{-4}$, which can be regarded as a measure for the ratio of the magnetic force to the gravity within the flux tube.

\section{STABILITY OF FLUX TUBE}

The background plasma is taken to be nearly neutrally stable, and hence the stratification satisfies $0<\gamma-(1+m) \ll 1$. The presence of a magnetic flux tube creates a small contrast in the temperature. Although the equilibrium magnetic fields possess axisymmetry, the equilibrium pressure does not. This complication makes an accurate evaluation of the stability criterion nontrivial. However, one may attack this stability problem by using the energy principle. The energy principle is usually a powerful tool for proving instabilities, and only in rare cases can it be successfully used to provide stability. In view of the complications arising from the asymmetric equilibrium, we shall limit our attentions to the most common instability in the $\beta \gg 1$ plasma, i.e., the localized instability (Freidberg 1982). Because of the locality, the instability tends not to be so disruptive as to destroy the entire flux tube. We will show that the free energy associated with the field-line twist in the outer layer can be sufficient to drive the MHD instability, and that when no twist is present the flux tube is stabilized by the stability of the overshoot region.

A general formulation of the potential energy, in the context of energy principle, for the MHD equilibrium in a gravitationally stratified plasma is, to our knowledge, not present in the literature. Hence, we shall now derive a useful form for the MHD potential energy, with which the stability of the flux-tube equilibrium can be addressed. The potential energy is constructed by taking an inner product of the perturbed force $\delta \boldsymbol{F}$ with the Lagrangian displacement of the fluid $\xi$. The primitive form of the potential energy so constructed can be written as

$$
\begin{aligned}
\delta^{2} W= & \frac{1}{2} \int d^{3} x\left[\frac{1}{4 \pi}(\delta \boldsymbol{B}-2 \pi \xi \times \boldsymbol{J})^{2}\right. \\
& -\pi(\xi \times \boldsymbol{J})^{2}+\gamma P(\nabla \cdot \xi)^{2}+(\nabla \cdot \xi) \\
& \times(\boldsymbol{\xi} \cdot \nabla P+\boldsymbol{\xi} \cdot \boldsymbol{g} \rho)+\boldsymbol{g} \cdot \boldsymbol{\xi} \boldsymbol{\xi} \cdot \nabla \rho],
\end{aligned}
$$

where $\boldsymbol{J} \equiv \boldsymbol{\nabla} \times \boldsymbol{B} / 4 \pi$ and the continuity equation, $\delta \rho=-(\xi \cdot \nabla \rho+\rho \nabla \cdot \xi)$, has been used. The magnetic perturbations can be written as $\delta \boldsymbol{B}=\boldsymbol{B} \cdot \nabla \boldsymbol{\xi}-\boldsymbol{\xi} \cdot \nabla \boldsymbol{B}-\boldsymbol{B} \nabla \cdot \boldsymbol{\xi}$, to be substituted into equation (9) for a complete description of the potential energy in terms of the displacement $\xi$.

In analogy to the derivation for convective instability of a nonmagnetized plasma, we shall first deal with the compressibility effects. Substituting $\delta \boldsymbol{B}$ into equation (9), we may first collect the terms containing $\nabla \cdot \xi$. Arrange these terms so that they are expressed as a combination of quadratures, and we have

$$
\begin{aligned}
\delta^{2} W_{c} \equiv & \frac{1}{2} \int d^{3} x\left(\frac{\boldsymbol{B}^{2}}{4 \pi}+\gamma P\right)\left\{\nabla \cdot \boldsymbol{\xi}+\frac{1}{\boldsymbol{B}^{2} / 4 \pi+\gamma P}\right. \\
& \left.\times\left[\boldsymbol{\xi} \cdot \nabla\left(P+\frac{\boldsymbol{B}^{2}}{8 \pi}\right)-\frac{\boldsymbol{B} \cdot(\boldsymbol{B} \cdot \nabla \boldsymbol{\xi})}{4 \pi}\right]\right\}^{2} \\
& -\frac{1}{2} \int \frac{d^{3} x}{\boldsymbol{B}^{2} / 4 \pi+\gamma P}\left[\boldsymbol{\xi} \cdot \nabla\left(P+\frac{\boldsymbol{B}^{2}}{8 \pi}\right)-\frac{\boldsymbol{B} \cdot(\boldsymbol{B} \cdot \nabla \boldsymbol{\xi})}{4 \pi}\right]^{2} .
\end{aligned}
$$

At this point, we may adopt a trial displacement that makes the first quadratic term in equation (10) vanish,

$$
\boldsymbol{\nabla} \cdot \boldsymbol{\xi}+\frac{1}{\boldsymbol{B}^{2} / 4 \pi+\gamma P}\left[\boldsymbol{\xi} \cdot \nabla\left(P+\frac{\boldsymbol{B}^{2}}{8 \pi}\right)-\frac{\boldsymbol{B} \cdot(\boldsymbol{B} \cdot \nabla \boldsymbol{\xi})}{4 \pi}\right]=0 \text {. }
$$

It fixes one component of the trial displacement. Since $P \gg B^{2} / 8 \pi$, this requirement for the displacement is almost the same as that of a stratified neutral fluid, i.e., a displacement that makes no pressure variation against the background. For the case under consideration where the variation length scale is much smaller than the background scale height by a factor $\epsilon$, such a condition is practically the incompressibility, $\boldsymbol{\nabla} \cdot \boldsymbol{\xi}=0$.

On the other hand, it is well-known that the unstable MHD modes tend to localize near the surfaces where $\boldsymbol{B} \cdot \boldsymbol{\nabla} \boldsymbol{\xi}_{\perp}=0$, the magnetic resonant surfaces, so as to avoid the stabilizing magnetic tension in a sheared magnetic field (Freidberg 1982). Here $\xi_{\perp}$ is the displacement across the flux surface. Realizing the physical significance of the magnetic resonance, one manages to isolate its contribution from the potential in equation (9). We collect the remaining terms arising from the Lorentz force, and express them in the following form:

$$
\begin{aligned}
\delta^{2} W_{b} \equiv & \int \frac{d^{3} x}{8 \pi}\left[(\boldsymbol{B} \cdot \nabla \boldsymbol{\xi}-\boldsymbol{\xi} \cdot \nabla \boldsymbol{B})^{2}\right. \\
& -(\boldsymbol{\xi} \times \nabla \times \boldsymbol{B}) \cdot(\boldsymbol{B} \cdot \nabla \boldsymbol{\xi}-\boldsymbol{\xi} \cdot \nabla \boldsymbol{B})] .
\end{aligned}
$$

The total $\delta^{2} W$ becomes

$$
\delta^{2} W=\delta^{2} W_{c}+\frac{1}{2} \int \boldsymbol{g} \cdot \boldsymbol{\xi} \xi \cdot \nabla \rho d^{3} x+\delta^{2} W_{b} .
$$

To proceed further, we shall take advantage of the small parameters $\beta^{-1}$ and $\epsilon$. To the leading order the fluid is unmagnetized, and only the one term associated with $P$ in the second quadrature of $\delta^{2} W_{c}$ (cf., equation [10]) and the second term of equation (13) are retained in $\delta^{2} W$. They have opposite signs and nearly cancel each other since the gas is nearly neutrally stable. To the first order $O\left(\beta^{-1}\right)$, the residue terms of $\delta^{2} W_{c}$ after the major cancellation by the second term of equation (13) become

$$
\begin{aligned}
\delta^{2} W_{1 c}= & \frac{1}{8 \pi} \int d^{3} x\left\{\left(\frac{\xi \cdot \nabla P_{0}}{\gamma P_{0}}\right)^{2}\left[B^{2}+4 \pi \gamma\left(\eta P_{0}+P_{1}\right)\right]\right. \\
& \left.+2 \frac{\xi \cdot \nabla P_{0}}{\gamma P_{0}}\left[\boldsymbol{B} \cdot(\boldsymbol{B} \cdot \nabla \boldsymbol{\xi})+\frac{\xi_{r} B_{\theta}^{2}}{r}\right]\right\}
\end{aligned}
$$


where $\eta \equiv(\gamma / 1+m)-1 \sim O\left(\beta^{-1}\right)$. An estimate of the magnitudes will place their relative importance in order. The first term on the right represents the stability of the overshoot region, which is at least of order $\epsilon^{2} B^{2}$ so as to ensure the stability of the main tube, and the second term is also of that order of magnitude; of the third term, $\boldsymbol{B} \cdot(\boldsymbol{B} \cdot \nabla \boldsymbol{\xi})$ $\left(\approx B_{y} B \cdot \nabla \xi_{y}\right)$ is of order $\epsilon B_{y} B_{\theta}$ but the $\xi_{r} B_{\theta}^{2} / r$ term is of order $\epsilon B_{\theta}^{2}$. As will be argued in the next section, a consistent dynamo picture requires $B_{\theta}=O\left(\epsilon B_{y}\right)$, yielding a negligibly small last term.

In the Appendix, we show how the following expression of $\delta^{2} W_{b}$ is obtained for the cylindrically symmetric equilibrium magnetic field:

$$
\begin{aligned}
\delta^{2} W_{b}= & \frac{1}{8 \pi} \int d^{3} x\left[\left(\boldsymbol{B} \cdot \nabla \xi_{\perp}\right)^{2}+\left(\boldsymbol{B} \cdot \nabla \xi_{y}\right)^{2}\right. \\
& \left.+\frac{B_{\theta}^{2}}{r}\left(\xi_{r}^{2}-\xi_{\theta}^{2}\right)-2 \frac{B_{\theta} B_{\theta}^{\prime}}{r} \xi_{r}^{2}\right]
\end{aligned}
$$

where $\xi_{\perp}=\xi-\xi_{y} \hat{y}$. Further manipulations of the potential energy involve explicitly eliminating $\xi_{y}$. This is because $\left|\partial \xi_{y} / \partial y\right| \ll\left|\partial \xi_{x} / \partial x\right| \sim\left|\partial \xi_{z} / \partial z\right|$, even for a $\xi_{y}$ comparable to or larger than the other two components, as a direct consequence of the very small gradient along the $y$ direction. Hence the almost-incompressibility condition of equation (11) imposes a constraint only on $\xi_{x}$ and $\xi_{z}$, with no concern to $\xi_{y}$. To collect all terms involving $\xi_{y}$, we move $\left(\boldsymbol{B} \cdot \nabla \xi_{y}\right)^{2}$ in $\delta^{2} W_{b}$ of equation (15) to $\delta W_{1 c}$ of equation (14), which is denoted as $\delta^{2} W_{2 c}$, and manage to form a quadrature:

$$
\begin{aligned}
\delta^{2} W_{2 c}= & \frac{1}{8 \pi} \int d^{3} x\left[\left(\boldsymbol{B} \cdot \nabla \xi_{y}-\frac{P_{0}^{\prime} B_{y}}{\gamma P_{0}} \xi_{z}\right)^{2}\right. \\
& \left.+4 \pi \gamma\left(\eta P_{0}+P_{1}\right)\left(\frac{P_{0}^{\prime}}{\gamma P_{0}}\right)^{2} \xi_{z}^{2}\right],
\end{aligned}
$$

where $P_{0}^{\prime} \equiv d P_{0} / d z$. The first positive quadrature can be made zero by requiring

$$
\xi_{z}=\frac{\gamma P_{0}}{B_{y} P_{0}^{\prime}} \boldsymbol{B} \cdot \nabla \xi_{y}
$$

Lastly, the incompressibility condition imposed by equation (11) demands that $\boldsymbol{\nabla} \cdot \boldsymbol{\xi}_{\perp} \approx 0$, and we hence let

$$
\boldsymbol{\xi}_{\perp}=\hat{\boldsymbol{y}} \times \nabla \psi
$$

where $\psi$ is a scalar field. The potential energy finally emerges as

$$
\begin{aligned}
\delta^{2} W= & \frac{1}{8 \pi} \int d^{3} x\left[\left(\boldsymbol{B} \cdot \nabla \frac{\partial \psi}{\partial r}\right)^{2}+\left(\frac{\boldsymbol{B}}{r} \cdot \nabla \frac{\partial \psi}{\partial \theta}\right)^{2}\right. \\
& -\frac{2 B_{\theta}}{r^{2}}\left(\frac{B_{\theta}}{r}\right)^{\prime}\left(\frac{\partial \psi}{\partial \theta}\right)^{2} \\
& \left.+\frac{1}{2 \gamma}\left(8 \pi \eta P_{0}-B_{y}^{2}\right)\left(\frac{P_{0}^{\prime}}{P_{0}}\right)^{2}\left(\frac{\partial \psi}{\partial x}\right)^{2}\right]
\end{aligned}
$$

where $B_{\theta}^{\prime} \equiv d B_{\theta} / d r$, and $\boldsymbol{B} \cdot \nabla \xi_{\perp}=\left(\boldsymbol{B} \cdot \nabla \xi_{r}\right) \hat{\boldsymbol{r}}+\left(\boldsymbol{B} \cdot \nabla \xi_{\theta}\right) \hat{\boldsymbol{\theta}}$ $+\left(B_{\theta} / r\right)\left(\xi_{r} \hat{\theta}-\xi_{\theta} \hat{\boldsymbol{r}}\right)$ and $P_{1} \approx-B_{y}^{2} / 8 \pi$ have also been used in equation (18).

Clearly, when a twist-free flux tube is to be stable, we need $8 \pi \eta P_{0} \geq B_{y}^{2}$, at the region where $B_{y}^{2}$ assumes the maximum value, which is at $r=0$ for the flux-tube equi- librium under consideration. Otherwise, the flux tube would undergo the Parker-like undular instability in its interior, which can be disruptive to the tube. (A simple way to illustrate such an instability is to adopt the planar geometry of a flux layer.) We shall thereafter assume the overshoot region to be sufficiently stably stratified so that $8 \pi \eta P_{0}>B_{y}(0)^{2}$. Also apparent in equation (18) is the additional stabilization by the presence of the field-line twist (i.e., a finite $B_{\theta}$ ), as long as $\left(B_{\theta} / r\right)^{\prime}<0$. Such twist-field stabilization has even been reported for dynamically evolving configurations (Dorch \& Nordlund 1998; Hughes et al. 1998). However, when an intense current density is present in the flux tube, i.e., $\left(B_{\theta} / r\right)^{\prime}>0$, it will provide a negative free energy to $\delta^{2} W$.

The current-drive negative energy concentrates within a thin outer layer, which may be regarded as a locally flat layer. We can take advantage of this flat-layer geometry to carry out a further analysis. Let $\psi$ be proportional to $\bar{\psi}(r) \exp \left(i k_{\chi} \chi+i k_{y} y\right)$, with $\chi$ being the coordinate along the local layer direction. Therefore the operator $\boldsymbol{B} \cdot \boldsymbol{\nabla}=i \boldsymbol{k} \cdot \boldsymbol{B}$. Because of the positive contribution from $\xi_{z}^{2}$ in equation (18), the $\chi$ component of the unstable perturbation tends to be perpendicular to $z$, i.e. in the $x$-direction, so that $\xi_{z}=-i k_{\chi} \bar{\psi}$ can be made smaller than $\xi_{x}=d \bar{\psi} / d r$. This situation occurs in the local layers on the top and bottom of the tube, where the potential energy becomes

$$
\begin{aligned}
\delta^{2} W= & \frac{1}{8 \pi} \int d^{3} x\left[(\boldsymbol{k} \cdot \boldsymbol{B})^{2}\left[\left(\left|\frac{d \bar{\psi}}{d r}\right|\right)^{2}+k_{\chi}^{2}|\bar{\psi}|^{2}\right]\right]-\frac{1}{8 \pi} \int d^{3} x \\
\times & {\left[2 B_{\theta}\left(B_{\theta} / r\right)^{\prime}-\frac{\gamma \epsilon^{2}}{2(\gamma-1)^{2}}\left(8 \pi \eta P_{0}-B_{y}^{2}\right)\right] k_{\chi}^{2}|\bar{\psi}|^{2} . }
\end{aligned}
$$

For a layer with a sufficiently intense current density $B_{\theta}^{\prime}$, the potential energy can become negative.

Quantitatively, the instability condition can be evaluated by considering the trial solution near the magnetic resonant surface, $\boldsymbol{k}\left(r_{m}\right) \cdot \boldsymbol{B}\left(r_{m}\right)=0$, which can be expanded by the leading Taylor series as $\left[(\boldsymbol{k} \cdot \boldsymbol{B})^{\prime}\right]^{2} X^{2}$, with $X \equiv \boldsymbol{r}-r_{m}$. The Euler-Lagrange equation near $r=r_{m}$ then becomes

$$
\frac{d^{2} \bar{\psi}}{d X^{2}}+\frac{Q}{4 X^{2}} \bar{\psi}=0
$$

where

$$
Q \equiv \frac{4 k_{\chi}^{2}}{\left[(\boldsymbol{k} \cdot \boldsymbol{B})^{\prime}\right]^{2}}\left[2 B_{\theta}\left(B_{\theta} / r\right)^{\prime}-\frac{\gamma \epsilon^{2}}{2(\gamma-1)^{2}}\left(8 \pi \eta P_{0}-B_{y}^{2}\right)\right] .
$$

When $Q \geq 1$, the trial solution $\bar{\psi}$ will become rapidly oscillatory, and it is well-known that this indicates instability (Freidberg 1982); otherwise, the system is stable. Note that the $k$ dependence in $Q$ suggests that the instability favors low- $k$ perturbations. However, since we have made a local approximation, $k_{\theta}$ cannot be too small, for example $k_{\chi}=$ $k_{\theta}=m / r \geq 4 / r$ or a wavelength equal to or smaller than $1 / 4$ of the layer circumference. Using the magnetic resonance condition, $k_{y}$ can be eliminated in favor of $k_{\chi}$, i.e., $\quad\left[(\boldsymbol{k} \cdot \boldsymbol{B})^{\prime}\right]^{2}=m^{2}\left[\left(B_{\theta} / B_{y}\right) B_{y}^{\prime}-\left(B_{\theta} / r\right)^{\prime}\right]^{2} . \quad$ Moreover, since $8 \pi \eta P_{0}-B_{y}^{2}(0)>0$, we may further parametrize $8 \pi \eta P_{0}-B_{y}^{2} \approx 8 \pi \eta P_{0} \equiv(1+\Delta) B_{y}^{2}(0)$ near the outer layer. 
The local instability condition therefore becomes

$$
\begin{aligned}
Q= & \left\{\frac{8}{r^{2}\left[\left(B_{\theta} / r B_{y}\right) B_{y}^{\prime}-\left(B_{\theta} / r\right)^{\prime}\right]^{2}}\right\} \\
& \times\left[B_{\theta}\left(B_{\theta} / r\right)^{\prime}-\frac{\gamma \epsilon^{2}(1+\Delta) B_{y}^{2}(0)}{4(\gamma-1)^{2}}\right]>1 .
\end{aligned}
$$

To yield a positive $Q$, we need a sufficiently large position $B_{\theta}^{\prime}$, which can occur when $\kappa$ in equation (5) is large. On the other hand, a smaller value of the denominator in the first factor of $Q$ in equation (22) helps yield a positive $Q$. We therefore look for the condition where $\left(B_{\theta} / B_{y}\right) B_{y}^{\prime}$ $-\left(B_{\theta} / r\right)^{\prime} \rightarrow 0$ from the field equilibrium given by equations (4) and (5). Apparently this is possible in the region where $r>a$ and the toroidal field has reversed the direction, because $\left(B_{\theta} / r\right)^{\prime}$ and $B_{\theta}$ are both positive and $B_{y}^{\prime}$ negative. We shall emphasize that the reversal of $B_{y}$ is critically needed to yield a value of $Q \geq 1$. If $B_{y}$ were to remain positive, the two terms in the denominator of $Q$ in equation (22) would have added and become much greater than the numerator $B_{\theta}\left(B_{\theta} / r\right)^{\prime}$ precisely in the region where $B_{\theta}^{\prime}$ is large and $Q$ positive; thus, it would not have been possible for the value of $Q$ to exceed unity, thereby giving a stable system.

In $\S 4.4$ we will consider the conservation of magnetic helicity and other constraints provided by the observed emerging flux to estimate the appropriate parameters for the equilibrium field given in equations (4) and (5). There these parameters are found to be $\kappa \approx 30, a \approx 3 / 4$, and $h \approx \epsilon \approx$ few $\times 10^{-2}$. Substituting $\gamma=5 / 3$, we find that with these magnetic field parameters, it requires the stabilizing $\Delta$ of the overshoot-region stratification to be in the range

$$
0<\Delta<1
$$

to ensure a positive $Q$ and still maintaining a stable main tube. The unstable magnetic resonant surfaces should also be distributed immediately outside the field-reversal layer around $r-a \sim 1 / 7$, within a radial range about $|\delta X| \sim 1 /$ 10 , to warrant a $Q>1$.

In sum, the current-drive instability can take place only on the top and bottom parts of a flux tube embedded in stably-stratified overshoot region, provided that the field configuration and the stabilizing stratification satisfies equation (22). For an equilibrium field given in equations (4) and (5), with the specific parameters determined in $\S 4.4$, we find that equation (23) is an approximate instability criterion.

\section{DISCUSSION}

In the section, we shall discuss how the above findings may fill some gaps in our understanding of the turbulent dynamo operative inside the Sun.

\subsection{Nonlinear Development of Instability}

As the embedded flux tube accumulates a sufficient amount poloidal field, an ideal MHD instability is likely to take place around the top and bottom of the tube. The nonlinear development is expected to be such that the turbulent motion tears apart the outer layer of the main tube through field-line reconnection (because this is the only way for the unstable system to remove the free energy). Since the dispatched flux should be hotter than it originally was due to magnetic dissipation, the disconnected flux rope is there- fore expected to float upward against the gravity and eventually emerges above the solar surface. Of course, the main tube may also receive a small part of the heat so generated. But the interior strong magnetic field serves to insulate the heat and moreover the main tube has a greater heat capacity weighed by its greater mass, and hence the buoyancy force density on the main tube is relatively weak.

To assess whether the buoyancy force may indeed be large enough to lift the dispatched flux into the convection zone, we shall estimate the amount of heat generated through the magnetic dissipation. From the linear analysis of potential energy given earlier, we know that an additional internal energy density of order $B_{\theta}^{2} / 8 \pi$ will suffice to lift up the unstable flux. Due to the reversed toroidal field, it is the toroidal field that should dominantly contribute to the magnetic reconnection in giving out the heat. The amount of toroidal field energy density to be dissipated is about $\left(B_{y}^{\prime} \delta X\right)^{2} / 8 \pi$, where $\delta X$ is the width of the layer, within which the reconnection takes place. On the other hand, we also have $B_{y}^{\prime} \sim O\left(\epsilon^{-1} B_{\theta}^{\prime}\right)$, implying that a reconnection layer width of order $\epsilon\left(\sim \mathrm{few} \times 10^{-2}\right)$ will be adequate. However, another estimate of the reconnection layer width, over which the unstable magnetic resonance surfaces spread, given in the end of $\S 3$, shows that $|\delta X| \sim 1 / 10$. Thus, the heat so generated should be sufficient to lift the unstable flux into the convection zone. From this estimate, we again witness the crucial need of the toroidal-field reversal for the present picture of flux emergence to work properly.

One expects that the unstable flux should organize itself into twisted flux ropes after the reconnection, and in the convection zone, the dynamics of the twisted flux ropes is highly nonlinear. Nonlinear developments of the disconnected twisted flux ropes have been demonstrated earlier in a simplified two-dimensional geometry where the outer layer of the flux tube is approximated by a planar flux layer (Cattaneo et al. 1990a), though the initiation of that instability is caused by the buoyancy force rather than the magnetic force purported here. Recently, the detailed three-dimensional dynamics of flux ropes in the highly nonlinear regime has also been reported (Fan et al. 1998b, 1999). We do expect our flux-rope evolution to be closely related to that reported in those more realistic threedimensional simulations.

To close this discussion, we shall briefly return to address the required nature of the MHD instability. One notes that to leave the main tube intact during the eruption of the outer layer, the MHD instability cannot be of global nature, nor can the instability be too strong. It appears that the local instability shown in $\S 3$ just suits such a requirement. That is, the instability is localized within a thin outer layer, thereby avoiding exerting a sizable perturbing force to the main tube. By contrast, the global $m=1$ kink mode should have a difficultt to become unstable. By contrast, the global $m=1$ kink mode should have a difficulty to become unstable. This is because it involves a displacement of the whole tube, but most of the tube interior is stabilized by the overshoot-region stratification when the displacement contains a vertical component, and also stabilized by the magnetic tension when the displacement is purely horizontal.

\subsection{Connections to the Solar Dynamo}

Since the erupted flux is expected to be detached from the main tube outside the surface where the field-line twist 
reverses, the ejected magnetic fields carry only one sign of magnetic helicity. The possibility of removing magnetic helicity from the dynamo region can have profound implications for the mean-field turbulent dynamo. This is because in order for the mean-field dynamo to operate it is necessary to break the conservation of magnetic helicity in the dynamo region.

A self-consistent, mean-field dynamo theory has recently been derived, and it was shown that generation of largescale magnetic fields is impossible in a closed volume of a highly conducting plasma, due primarily to the conservation of magnetic helicity (Gruzinov \& Diamond 1994). However, we shall point out that if the field lines are allowed to escape from the region where the dynamo operates, the magnetic helicity in the dynamo region may no longer conserve. In the present model, helical field lines with a definite sign are expected to be expelled from the dynamo flux tube, and therefore the magnetic helicity in it must not conserve. This flux expulsion mechanism can therefore circumvents the difficulty faced by the mean-field turbulent dynamo advocated previously (Cattaneo \& Vainshtein 1991; Cattaneo 1994; Gruzinov \& Diamond 1994). In this connection, one is led to speculate the active participation of the flux expulsion for the operation of solar dynamo, apart from the conventional notions of "twist," "fold," and "break" motions of the field lines.

From another perspective in considering the feedback of the Lorentz force to the kinematic dynamo, Cattaneo \& Vainshtein (1991) and Cattaneo (1994) have argued that owing to a similar mechanism shown by Zeldovich (1957) for two-dimensional MHD, one expects that in three dimensions the small-scale magnetic fields must also overwhelm the large-scale magnetic fields. The former will have to distort the fluid turbulence to such an extent that the latter has no chance to grow. They also give an independent estimate for the saturated strength of the large-scale fields, which turns out to be the same as that given by Gruzinov \& Diamond (1994). Again, their arguments assume the MHD system to be a closed system. In such a closed system, the turbulent flow becomes so distorted by the small-scale fields that the magnetic field lines stop undergoing random walks, and a long-term phase lock between the field lines and the flows can be established (Cattaneo 1994). However, in an open system, the magnetic flux can reside within the dynamo active region in a finite time interval, and the about-to-be established phase correlations between the flow and the field can be removed along with the escaped magnetic flux. Once the memories are lost, cascades of magnetic fields from the small scales toward the large scales will become permissible. Inverse cascades or three-dimensional, helical MHD turbulence are of this nature, and they have been investigated extensively in the past using a turbulence closure scheme (Pouquet, Frisch, \& Leorat 1976).

\subsection{Signs of Magnetic Helicity in Erupted Flux Ropes}

As the emerging flux possesses a finite twist, we now turn to exploring the sign of the twist, which is in principle a measurable quantity. To this aim, we must first briefly review the essence of the "dynamo quench" theorem given by Gruzinov \& Diamond (1994). For a linear $\alpha$-dynamo, the quantity $\alpha(\propto$ amplification rate) is proportion to the fluid helicity density $h_{v}$, where $h_{v} \equiv\langle\boldsymbol{v} \cdot \boldsymbol{\nabla} \times \boldsymbol{v}\rangle$ and the plasma density has been normalized to unity (Krause \& Steenbeck 1967; Radler 1969). However, for a self-consistent nonlinear dynamo, it can be shown that

$$
\alpha \propto h_{v}-h_{j}
$$

(Pouquet et al. 1976; Gruzinov \& Diamond 1994), where the current helicity density $h_{j} \equiv\langle\delta \boldsymbol{B} \cdot \nabla \times \delta \boldsymbol{B}\rangle / 4 \pi$. In the self-consistent dynamo theory of Gruzinov $\&$ Diamond, the fluid helicity $h_{v}$ is shown to be almost completely canceled by the current helicity $h_{j}$, as a result of the conservation of magnetic helicity. This cancellation is a manifestation of the fact that the angular momentum flux of the small-scale fluid motion imparted into the magnetic field is opposed by that of the small-scale magnetic field into the fluid. Thus, the turbulent flow motion becomes aligned with the turbulent magnetic field and hence the fluid exerts no net torque to the fields for generating the large-scale field. Consequently, in order to avoid the otherwise nearly complete cancellation of $h_{v}$ by $h_{j}$ and to allow for the mean-field dynamo to operate, it is essential to remove a part of the current helicity density $h_{j}$ of the same sign as $h_{v}$ in the dynamo region. The flux expulsion described above can just serve this purpose. Note that a finite current helicity density implies a finite magnetic helicity density $\langle\delta \boldsymbol{A} \cdot \delta \boldsymbol{B}\rangle$, where $\delta \boldsymbol{A}$ is the fluctuating vector potential, and hence the removal of current helicity implies the removal of magnetic helicity.

To proceed further on exploring the sign of the field-line twist, we note that in the bottom of the convection zone, the pseudoscalar $h_{v}$ is driven primarily by the pseudoscalar $\boldsymbol{g} \cdot \boldsymbol{\Omega}$ where $\boldsymbol{\Omega}$ is the solar rotation frequency. This can be understood by examining the vertical component of the vorticity equation:

$$
\frac{D \omega_{z}}{D t}+(\nabla \cdot v) \omega_{z}-\omega \cdot \nabla v_{z}=-2(\nabla \cdot v) \Omega_{z}+2 \Omega \cdot \nabla v_{z},
$$

where the vorticity $\omega \equiv \nabla \times v$ and $D / D t$ is the total time derivative. The driving source for fluid vorticity becomes clear in the limit where the gradient of $v_{z}$ is small and the last terms on both sides of equation (25) are negligibly small. The amplification rate of $\omega_{z}$ is proportional to the compression, $c \equiv-\nabla \cdot v$, and the driving source proportional to $c \Omega_{z}$. The downdraft motion is compressed (i.e., $c>0$ ), and hence should carry an amplified vertical vorticity of the same sign as $\Omega_{z}$. Indeed, recent threedimensional simulations have demonstrated that the downdraft flows do carry much more intense vorticity than the uplifting plumes in the convection zone (Brummell, Hurbert, \& Toomre 1996); moreover, the helical downdraft can pump current helicity of the same sign as the fluid helicity into the magnetic field (Tobias et al. 1998). The fluid helicity $\left(\sim \omega_{z} v_{z}\right)$ of the downdraft should therefore have the same sign as $g \cdot \boldsymbol{\Omega}$. Such a downdraft provides the needed fluid helicity injected into the overshoot region to generate a finite $\alpha$ for the kinematic dynamo. The injection of fluid angular momentum into the magnetic fields continues until the small-scale fields are aligned with the flow. At this stage, no torque can be exerted from the flow to the magnetic field, the current helicity $h_{j}$ becomes as large as $h_{v}$, and the dynamo thus ceases to operate.

Such a physical picture of "dynamo quench" can be invalidated if the helical fields are continually removed from the dynamo region once the field-line twist exceeds some 
threshold strength as demonstrated in $\S 3$. Therefore the turbulent flows can subtend a finite angle from the smallscale fields and exert a finite torque to the field lines. The removed current helicity $h_{j}$ must possess the same sign as the fluid helicity $h_{v}$, whose sign is in turn the same as $\boldsymbol{g} \cdot \boldsymbol{\Omega}$. In fact, for the dynamo to operate, the amount of current helicity to be removed needs only be a small fraction $\delta h$ of the fluid helicity $h_{v}$, as long as $\delta h / h_{v} \gg R_{m}^{-1}$. However, for the following reasons, $\delta h / h_{v}$ is likely to be on the order of unity and the dynamo growth rate can resume a magnitude comparable to the kinematic dynamo growth rate. This is because (1) the observed rate of emergence flux is sizable, $10^{22} \mathrm{Mx}$ in few months, (2) these magnetic flux contains twist field lines, and (3) more importantly, recent observations show that these emerging fields statistically have a definite sign of helicity in each hemisphere (Pevtsov, Canfield, \& Metcalfe 1994, 1995). It follows that a substantial fraction of helicity injected by the helical downdraft and accumulated in the outer layer of flux tube is continually removed, therefore yielding $\delta h / h_{v} \sim O(1)$ and $h_{v}-h_{j} \sim$ $O\left(h_{v}\right)$. In addition, according to the sign rule, we can make a definite prediction that the emerging flux should carry a current helicity of the same sign as $g \cdot \nabla$. That is, the twists of the emerging field lines should obey the left-hand rule in the northern hemisphere and right-hand rule in the southern hemisphere, which agrees with the observations (Pevtsov et al. 1994, 1995). This prediction holds regardless of the different phases of the solar cycles since $\boldsymbol{g} \cdot \boldsymbol{\Omega}$ does not change with solar cycles.

\subsection{Estimation for Physical Parameters in the Dynamo Flux Tubes}

Having discussed the sign of current helicity to be removed, we now turn to a quantitative discussion on the amount of magnetic helicity to be removed from the dynamo flux tube, from which the physical parameters of the dynamo flux tube can be estimated. For a flux rope about to leave the overshoot region, it should have a strength as large as $10^{5} \mathrm{G}$, as suggested by Caligari et al. (1995). The field energy exceeds the turbulence kinetic energy by two orders of magnitude, and hence the depth, into which the helical downdraft can exert a torque, should be small. This point has also been addressed in $\S 4.1$ from another perspective where we discussed the nonlinear development of the instability. That is, the quantity $a$ defined in equation (5) should satisfy $1-a \ll 1$. Though the exact value of $a$ is determined by the strength of the helical downdraft and the differential rotation, which are model dependent and rather uncertain, we now present another line of arguments for its evaluation.

The observations show that the lifetime of the largest active region is about few months (Golum et al. 1981) but the time scale for the dynamo growth is believed to be about few years. If the disruption of a large active region on the solar surface physically corresponds to the elimination of magnetic flux, it follows that the above two time scales can only be reconciled if the dispatched flux rope has a magnetic flux about $10 \%$ of that contained in the main dynamo tube. Since the magnetic flux of a large active region amounts to about $10^{22} \mathrm{Mx}$ (Caligari et al. 1995), it follows that the dynamo flux tube should contain a magnetic flux about $10^{23} \mathrm{Mx}$. On the other hand, an emerging flux rope should have a field strength about $10^{5} \mathrm{G}$ in the overshoot region in order for it to retain its integrity upon emerging to the solar surface (Choudhuri \& Gilman 1987; Caligari et al. 1995); the initial radius of the emerging flux rope therefore has a minor radius about $2000 \mathrm{~km}$. Geometrically, we may use the approximate equality for the cross sections of the flux rope after reconnection and the unstable flux layer before the reconnection to estimate the unstable layer width. From the two conditions - the average field strength outside $a r_{\text {tube }}$ being $10^{5} \mathrm{G}$ and the total flux within $r_{\text {tube }}$ being $10^{23} \mathrm{Mx}$, one may adopt equation (4) to solve for the two unknowns, $r_{\text {tube }}$ and $a$. A straightforward algebra shows that the fractional thickness $a \sim 0.75$, and that the dynamo flux tube radius $r_{\text {tube }} \sim 3000 \mathrm{~km}$. It also yields peak toroidal field strength at the tube core about $10^{6} \mathrm{G}$ and the average toroidal field strength about $5 \times 10^{5} \mathrm{G}$ in the main tube.

Furthermore, from the surface observation of the sunspot magnetic field, the average longitudinal field is about 1000 $\mathrm{G}$ and the transverse field about few $\times 10^{2} \mathrm{G}$. The $1000 \mathrm{G}$ strength requires the cross section of the erupted flux rope to expand by 2 orders of magnitude according to the conservation of toroidal magnetic flux, giving a reasonable spot diameter about $2 \times 10^{4} \mathrm{~km}$. If the poloidal magnetic flux is also conserved, $B_{p}\left(\propto r^{-1}\right)$ decreases only by one order of magnitude when the flux tube emerges to the surface. It follows that the average poloidal field strength of the flux rope immediately after dispatched from the main tube is about $f e w \times 10^{3} \mathrm{G}$, suggesting that outside the radius $a$ of the dynamo flux tube, the average $B_{p}$ is also about few $\times 10^{3} \mathrm{G}$ and the average $B_{p} / B_{y} \sim f e w \times 10^{-2}$ in the outer layer. This ratio is close to the magnitude of $\epsilon$ given in the end of $\S 2$, and therefore justifies our previous ordering that $\epsilon B_{y} \sim O\left(B_{y}\right)$, or $\epsilon \sim h$ adopted in $\S 3$.

In order to further determine the parameter $\kappa$ in equation (5), which characterizes the strength of $B_{p}$ in the tube outer layer relative to that in the tube interior, we turn to the consideration for the conservation of magnetic helicity. The magnetic helicity can be shown to be proportional to $N \Psi^{2}$ (Zeldovich, Ruzmaikin, \& Sokolov 1984; Chiueh 1997), where $\Psi$ is the total toroidal magnetic flux and $N$ is the average number of poloidal twists along a field line around the torus. The twist number $N$ can be conveniently expressed as

$$
N=\frac{\left\langle B_{p}\right\rangle R}{\left\langle B_{y}\right\rangle r_{\text {tube }}},
$$

with $R$ being the major radius of the torus. The twist number is a conserved quantity if the toroidal magnetic flux in the tube does not change.

To maintain a zero total magnetic helicity, the magnetic helicity retained in the main flux tube should have an opposite sign from that ejected, but with a magnitude equal to the sum of those fields emerged in the past in a single cycle. This consideration is correct provided that the flux tubes in both hemispheres do not annihilate in the solar interior, and we shall assume so. Thus, we have the maintube helicity

$$
N \Psi^{2}=-\sum_{i} N_{i} \Psi_{i}^{2},
$$

where the subscript $i$ stands for the index of each escaped flux rope. In fact, we have previously used $\Psi \sim \sum_{i} \Psi_{i}$ to estimate the total magnetic flux within the main tube and found that $\left\langle\Psi_{i}\right\rangle \sim \Psi / 10$. It follows from equation (27) that $N \sim\left\langle N_{i}\right\rangle / 10$. Equation (26), along with the estimates that $\left\langle B_{y}\right\rangle \sim$ several $\times 10^{5} \mathrm{G}$ in the main tube and $B_{y} \sim 10^{5} \mathrm{G}$ in 
the dispatched flux rope give $\kappa \sim 30$, when the $B_{p}$ profile in equation (5) is adopted. It also yields that the average $B_{p}$ within the main tube can only be as low as $10^{3} \mathrm{G}$. Such a value of $\kappa$ has been used to show the current-drive MHD instability in $\S 3$.

\subsection{Global Evolution of Magnetic Helicity}

Having the above results, we may now give an overall picture as to how the magnetic helicity should evolve. Since the magnetic flux of one-sign helicity is continually ejected out of the dynamo flux tube, the magnetic helicity of the other sign must therefore accumulate in the main tube. If the magnetic fields in both hemispheres are unconnected, the total magnetic helicity in each hemisphere must separately conserve. In addition, if the initial magnetic helicity is zero at the start of each half solar cycle, the main tube will have to accumulate all the opposite magnetic helicity lost to the solar atmosphere as the solar cycle proceeds. This important property has been used to evaluate $\kappa$ earlier.

Where does the magnetic helicity go? Observationally, it has been found that during the coronal-mass-ejection events the ejected plasmoids contain helical field lines, and this has led to a suggestion that interplanetary space is the ultimate destination for the magnetic helicity expelled from the solar interior (Low 1996 1998, private communication). On the other hand, the magnetic helicity accumulated in the dynamo flux tube cannot continue throughout the entire solar cycle. At the end of each half cycle, the flux tubes in both hemispheres drift towards the equator, but the convective turbulence near the equator contains far less helicity, as $\boldsymbol{\Omega} \cdot \boldsymbol{g} \rightarrow 0$. This substantially weakens the dynamo growth at the end of the half cycle. Moreover, the two dynamo flux tubes eventually merge together and annihilate at the equator at the end of the half cycle. The global electromagnetism of the Sun then has to go back to the starting point with a zero magnetic helicity, poised to start a new halfcycle at higher latitudes where $\boldsymbol{\Omega} \cdot \boldsymbol{g}$ is finite.

\subsection{Comparisons with Observations}

Apart from the evidence of field-line twists in the coronal mass ejecta, one may hope to directly measure the three components of the solar surface fields, by the Zeeman effects, to determine the (current) helicity of the emerging flux. Unfortunately, the vectorial nature of the transverse magnetic fields is notorious for suffering from an $180^{\circ}$ ambiguity (Sakurai 1989) in the polarization measurements, and therefore the measurement for the sign of field-line twists is intrinsically rather uncertain. Nonetheless, some techniques have been developed to infer the direction of the transverse field (Gary et al. 1987), and they generally work better for coherent large sunspots (Chang et al. 1996). Recently, Pevtsov et al. $(1994,1995)$ have managed to determine the averaged current helicity in the active regions. Magnetic field data from 1990 to 1994 (within the same solar cycle 22) are used for such an analysis, and they have found that the averaged magnetic helicity indeed shows the same trend as what this work predicts, i.e., the left-hand rule in the northern hemisphere and right-hand rule in the southern hemisphere. Unfortunately, these measurements are difficult to conduct, in the face of the $180^{\circ}$ ambiguity in the transverse fields, and results of these measurements can at best be statistical which are plagued with large scatters in the data. In fact, one would like to make a stronger statement about the sense of field-line twist in the emerging flux than the statistical one. As mentioned above, determination of the field-line twist is a much easier task for the coherent large sunspots than for the average active-region fields. Hence the prediction of our dynamo scenario should be best tested against the sunspot fields. In fact it is now timely to compare the sunspot fields in cycle 22 and cycle 23 and to test whether the magnetic helicity indeed remains unchanged in different cycles.

\section{CONCLUSION}

How well an intense magnetic field can be stored in the interior of the Sun is an issue that ultimately determines whether the solar dynamo may operate. Although it has been pointed out that a flux tube should be slightly cooled in order to satisfy pressure balance with the background plasma (Moreno-Insertis \& Ferriz-Mas 1992; Ferriz-Mas 1996), such a flux tube should also be slightly twisted in order to enhance the field self-confinement when it erupts (Moreno-Insertis \& Emonet 1996; Emonet \& MorenoInsertis 1998; Dorch \& Nordlund 1998; Hughes et al. 1998; Fan et al. 1998; Fan et al. 1998b, 1999). Much like the early work of Cattaneo et al. (1990a), we show that if the twist profile is in such a way that the interior of the flux tube has regions of opposite twists and contains a sufficient amount of toroidal current, the flux tube can be subject to a localized instability in the outer layer of the tube. In the nonlinear stage, the outer layer with only one sense of twist gets erupted, leaving the main tube intact with another sense of twist.

This picture is further strengthened by the recent observations that the emerging magnetic flux in the northern hemisphere has left-handed helicity and that in the southern hemisphere right-handed helicity (Pevtsov et al. 1994, 1995). Since the magnetic helicity is conserved, if the field lines in both hemispheres are not connected, it then follows that there must exist some magnetic flux in the interior of the Sun to account for the opposite magnetic helicity left behind. We propose that it is the toroidal field reversing the direction that gives rise to the desired opposite twists, as a consequence of the strong shear flow in the overshoot region. This proposition gains further supports from two different perspectives. First, in our stability analysis, we show that the current-driven MHD instability cannot take place without the presence of a reversed toroidal field. Second, during the nonlinear developments of unstable flux, we demonstrate that only the dissipation of the reversed toroidal field can a sufficient amount of heat be generated for the unstable flux to emerge into the convection zone.

For the model flux tube equilibrium given in equations (4) and (5), we have used the timescale argument to estimate that the dynamo tube has an average toroidal field about $5 \times 10^{5} \mathrm{G}$, with a tube radius about $3000 \mathrm{~km}$, and the field reversal occurs at about $2250 \mathrm{~km}$ from the tube center. From the sunspot observations and the conservation magnetic helicity, we also estimate that the poloidal field has a peak strength about several $\times 10^{3} \mathrm{G}$ and is largely confined within a thin layer centering around $2800 \mathrm{~km}$ from the tube center; in addition, there exists another smooth $B_{p}$ component, which is distributed across the flux tube with an average strength about $1000 \mathrm{G}$.

In the nonlinear regime of the localized instability, the unstable outer layer of the flux tube is expected to get peeled off and erupted. As the erupted field lines carry a definite sense of twist, this eruption mechanism can be effective in 
removing one sign of magnetic helicity away from the dynamo flux tube and leaving the other sign of helicity accumulated within the tube. This flux expulsion mechanism can break the conservation of magnetic helicity in the dynamo region, and thus circumvents the difficulty of the $\alpha$ quench. The present model further suggests that the dynamo $\alpha$ can resume a finite value comparable to that of the kinematic dynamo.

Even if this scenario is finally proven to be correct, there are still important questions that remain unanswered. Namely, why the solar dynamo should appear periodic, what makes the polarity of magnetic fields change in a half cycle, and why a new half cycle always starts below the $40^{\circ}$ latitudes? These are the ultimate issues a comprehensive dynamo theory will need to confront. Finally, we note that Parker (1993) has suggested that the $\alpha-\Omega$ dynamo may be nonlocal, in that the operation of the $\alpha$ mechanism can extend into the convection zone, whereas the $\Omega$ mechanism remains in the overshoot region. The present scenario that brings the flux emergence into the overall picture of dynamo action indeed involves a nonlocal effect in order to give a nonvanishing $\alpha$, and it does seem to point to the same direction put forth by Parker. A recent parametric study has demonstrated that such an idea of nonlocal kinematic dynamo, in fact leads to some desirable features of solar dynamo within a restricted parameter range (MacGregor \& Charbonneau 1997).

The author acknowledges stimulating discussions with B.C. Low on the subject of solar magnetic helicity. This work is supported in part by the National Science Council of Taiwan, under the grants NSC88-2112-M008-039.

\section{APPENDIX \\ REDUCED FORM OF $\delta^{2} W_{b}$}

The reduced form of $\delta^{2} W_{b}$ in equation (15) is obtained after some lengthy algebra, and we present the key steps for arriving at this result. Some preliminary identities are given below to facilitate the subsequent calculations:

$$
\begin{gathered}
\boldsymbol{B} \cdot \nabla \xi=\left[\boldsymbol{B} \cdot \nabla \xi_{r} \hat{\boldsymbol{r}}+\left(\boldsymbol{B} \cdot \nabla \xi_{\theta}\right) \hat{\boldsymbol{\theta}}+\left(\boldsymbol{B} \cdot \nabla \xi_{y}\right) \hat{y}\right]+\frac{B_{\theta}}{r}\left(\xi_{r} \hat{\theta}-\xi_{\theta} \hat{r}\right), \\
\boldsymbol{\xi} \cdot \nabla \boldsymbol{B}=\xi_{r} \boldsymbol{B}^{\prime}-\frac{\xi_{\theta} B_{\theta}}{r} \hat{\boldsymbol{r}},
\end{gathered}
$$

and

$$
(\nabla \boldsymbol{B}) \cdot \boldsymbol{\xi}=\left(\boldsymbol{\xi} \cdot \boldsymbol{B}^{\prime}\right) \hat{\boldsymbol{r}}-\frac{\xi_{r} B_{\theta}}{r} \hat{\boldsymbol{\theta}} .
$$

The magnetic energy density $\delta^{2} w$ can be reexpressed from its original form as

$$
(8 \pi) \delta^{2} w_{b}=|\boldsymbol{B} \cdot \nabla \boldsymbol{\xi}|^{2}-(\boldsymbol{B} \cdot \nabla \boldsymbol{\xi}) \cdot[(\nabla \boldsymbol{B}) \cdot \boldsymbol{\xi}+\boldsymbol{\xi} \cdot \nabla \boldsymbol{B}]+(\boldsymbol{\xi} \cdot \nabla \boldsymbol{B}) \cdot(\nabla \boldsymbol{B} \cdot \boldsymbol{\xi}) .
$$

Attention will now be paid to the second term on the right of equation (A4). Let us first focus on the contribution from the bracket of equation (A1) to this term. Taking advantage of the fact that $B$ is cylindrically symmetric and possesses only $\theta$ and $y$ components, we can have the equalities, $\boldsymbol{B} \cdot \nabla A=0$, where $A$ can be $d B_{y} / d r, d B_{\theta} / d r, B_{\theta}$, and $B_{y}$. Using these equalities, we find that the contribution in question can be expressed as the total derivatives with respect to $\theta$ and $y$, and hence they become a surface term of the potential energy and are dropped out from the consideration. The rest of the second term on the right of equation (A4) becomes the sum of $\left(B_{\theta} / r^{2}\right)\left[\left(\xi_{r}^{2}-\xi_{\theta}^{2}\right) B_{\theta}-\xi_{r}^{2} B_{\theta}^{\prime} r\right]$ and $\left(\xi_{\theta} B_{\theta} / r\right) \xi \cdot B^{\prime}$, where the prime stands for the radial derivative. The latter is found to be canceled by a same term from the last term on the right of equation (A4). The final magnetic potential energy turns out to assume a rather simple form:

$$
8 \dot{\pi} \delta^{2} w=(\boldsymbol{B} \cdot \nabla \xi)^{2}+\frac{B_{\theta}^{2}}{t}\left(\xi_{r}^{2}-\xi_{\theta}^{2}\right)-2 \frac{B_{\theta} B_{\theta}^{\prime}}{r} \xi_{r}^{2},
$$

or when the first term on the right is expanded using equation (A1),

$$
8 \pi \delta^{2} w=\left(\boldsymbol{B} \cdot \nabla \xi_{y}\right)^{2}+\left(\boldsymbol{B} \cdot \nabla \xi_{r}\right)^{2}+\left(\boldsymbol{B} \cdot \nabla \xi_{\theta}\right)^{2}+2\left(\frac{B_{\theta}^{2}}{r^{2}}-\frac{B_{\theta} B_{\theta}^{\prime}}{r}\right) \xi_{r}^{2}
$$

\section{REFERENCES}

Brummell, N. H., Hurbert, N. E., \& Toomre, J. 1996, ApJ, 473, 494 Caligari, P., Moreno-Insertis, F., \& Schussler, M. 1995, ApJ, 441, 886 Cattaneo, F. 1994, ApJ, 434, 200

Cattaneo, F., Chiueh, T., \& Hughes, D. 1990a, MNRAS, 247, 6p . 1990b, J. Fluid Mech., 219, 1

Cattaneo, F., \& Vainshtein, S. I. 1991, ApJ, 376, L21

Chang, K. L., Chiueh, T., Li, H. H., \& Tsay, W. S. 1996, Chinese J. Phys., 34,1

Chiueh, T. 1997, Chinese J. Phys., 35, 4
Choudhuri, A. R., \& Gilman, P. A. 1987, ApJ, 316, 788

D’Silva, S., \& Choudhuri, A. R. 1993, A\&A, 272, 621

Dorch, S. B. F., \& Nordlund, A. 1998, A\&A, 492, 329

Emonet, T., \& Moreno-Insertis, F. 1998, ApJ, 492, 804

Fan, Y., Fisher, G. H., \& Deluca, E. E. 1993, ApJ, 405, 390

Fan, Y., Zweibel, E. G., \& Lantz, S. R. 1998a, ApJ, 493, 480

Fan, Y., Zweibel, E. G., Linton, M. G., \& Fisher, G. H. 1998b, ApJ, 505, L59 
Ferriz-Mas, A. 1996, ApJ, 458, 802

Freidberg, J. P. 1982, Rev. Mod. Phys., 54, 801

Galloway, D. J., \& Weiss, N. O. 1981, ApJ, 243, 645

Golum, L., Rosner, R., Vaiana, G. S., \& Weiss, N. O. 1981, ApJ, 243, 309

Gruzinov, A. V., \& Diamond, P. H. 1994, Phys. Rev. Phys., 72, 1651

Gary, G. A., Moore, R. L., Hagyard, M. J., \& Haisch 1987, ApJ, 314, 782

Hughes, D. W., Falle, S. A. E. G., \& Joarder, P. 1998, MNRAS, 298, 433

Hughes, D. W., \& Proctor, M. R. E. 1988, Ann. Rev. Fluid. Mech., 20, 187

Kosovichev, A. G., et al. 1997, Sol. Phys., 170, 43

Krause, F., \& Steenbeck, M. 1967, Z. Naturforsch., 22a, 671

Low, B. C. 1996, Sol. Phys. 167, 217

MacGregor, K. B., \& Charbonneau, P. 1997, ApJ, 486, 484

Moffatt, H. K. 1978, Magnetic Field Generation in Electrically Conducting Fluids (Cambridge: Cambridge Univ. Press)

Moreno-Insertis, F., \& Emonet, T. 1996, ApJ, 472, 53

Moreno-Insertis, F., \& Ferriz-Mas, A. 1992, A\&A, 264, 686

Moreno-Insertis, F., Schussler, M., \& Ferriz-Mas, A. 1992, A\&A, 264, 686

Noyes, R. W., Weiss, N. O., \& Vaughan, A. H. 1984, ApJ, 287, 769
Parker, E. N. 1979, Cosmic Magnetic Fields (Oxford: Clarendon Press) 1993, ApJ, 408, 707

Pevtsov, A. A., Canfield, R. C., \& Metcalf, T. R. 1994, ApJ, 425, L117 1995, ApJ, 440, L109

Pouquet, A., Frisch, U., \& Leorat, J. 1976, J. Fluid Mech., 77, 321

Radler, K.-H. 1969, Monats. Dt. Akad. Wiss., 11, 194

Sakurai, T. 1989, Space Sci. Rev., 51, 11

Schmitt, J. H. M. M., Rosner, R., \& Bohn, H.-U. 1984, ApJ, 282, 316

Schussler, M., Caligari, P., Ferriz-Mas, A., \& Moreno-Insertis, F. 1994, A\&A, 281, L69

Spiegel, E. A., \& Weiss, N. O. 1980, Nature, 287, 616

Spruit, H. C. 1981, Astron. Astrophys., 98, 155

Tobias, S. M., Brummell, N. H., Clune, T. L., \& Toomre, J. 1998, ApJ, 502, L177

van Ballegooijen, A. A. 1982, A\&A, 113, 99

Zeldovich, Y. B. 1957, Sov. Phys. JETP, 4, 460

Zeldovich, Y. B., Ruzmaikin, A. A., \& Sokolov, D. D. 1984, Magnetic Field in Astrophysics (New York: Gordon \& Breach) 